\title{
Constraining Ceres' interior from its rotational motion
}

\author{
N. Rambaux ${ }^{1,2}$, J. Castillo-Rogez ${ }^{3}$, V. Dehant ${ }^{4}$, and P. Kuchynka ${ }^{2,3}$ \\ 1 Université Pierre et Marie Curie, UPMC-Paris 06, France \\ e-mail: [nicolas.rambaux; kuchynka]@imcce.fr \\ 2 IMCCE, Observatoire de Paris, CNRS UMR 8028, 77 avenue Denfert-Rochereau, 75014 Paris, France \\ 3 Jet Propulsion Laboratory, Caltech, Pasadena, USA \\ e-mail: julie.c.castillo@jpl.nasa.gov \\ ${ }^{4}$ Royal Observatory of Belgium, 3 avenue Circulaire, 1180 Brussels, Belgium
}

Received 24 January 2011 / Accepted 14 July 2011

\begin{abstract}
Context. Ceres is the most massive body of the asteroid belt and contains about $25 \mathrm{wt} \%$ (weight percent) of water. Understanding its thermal evolution and assessing its current state are major goals of the Dawn mission. Constraints on its internal structure can be inferred from various types of observations. In particular, detailed knowledge of the rotational motion can help constrain the mass distribution inside the body, which in turn can lead to information about its geophysical history.

Aims. We investigate the signature of internal processes on Ceres rotational motion and discuss future measurements that can possibly be performed by the spacecraft Dawn and will help to constrain Ceres' internal structure.

Methods. We compute the polar motion, precession-nutation, and length-of-day variations. We estimate the amplitudes of the rigid and non-rigid responses for these various motions for models of Ceres' interior constrained by shape data and surface properties.

Results. As a general result, the amplitudes of oscillations in the rotation appear to be small, and their determination from spaceborne techniques will be challenging. For example, the amplitudes of the semi-annual and annual nutations are around $\sim 364$ and $\sim 140$ milliarcseconds, and they show little variation within the parametric space of interior models envisioned for Ceres.

Conclusions. Owing to the small amplitudes of the nutation and the very long-period of the precession motion, the measurements of the rotational variations will be challenging to obtain. We also estimate the timescale for Ceres' orientation to relax to a generalized Cassini state, and find that the tidal dissipation within that object has probably been too small to drive any significant damping of its obliquity since formation. However, combining the shape and gravity observations of Dawn offers the prospect to identify departures of non-hydrostaticity on both global and regional scales, which will be instrumental in constraining Ceres' past and current thermal state. We also discuss the existence of a possible Chandler mode in the rotational motion of Ceres, whose potential excitation by endogenic and/or exogenic processes may help us to detect the presence of liquid reservoirs within the asteroid.
\end{abstract}

Key words. planets and satellites: dynamical evolution and stability - celestial mechanics

\section{Introduction}

The Dawn spacecraft will encounter and be inserted in orbit around Ceres in 2015. During the nominal mission, the asteroid will be mapped with high-resolution imaging at visual and infra-red wavelengths (VIR instrument), and its gravity field will be determined to the tenth degree and order (Konopliv et al. 2011; McCord et al. 2011). A major goal of the Dawn mission is to constrain the internal structure of Ceres, and in particular quantify the extent of differentiation, thus internal evolution that has been experienced by this dwarf planet (Russell et al. 2007; McCord et al. 2011).

While the primary objective of the high-resolution camera VIR is to map the surface composition and features of Ceres, an interesting contribution of these observations will be to characterize Ceres' rotational motion. Rotation properties have been shown to provide important constraints on the interiors of planetary bodies, (e.g., Mathews et al. 2002; Koot et al. 2010; Williams et al. 2001; Margot et al. 2007; Dehant et al. 2009) because they depend on both their mass distributions and viscoelastic properties. The departures from a uniform rotation and changes in the orientation of a body are indeed responses to an external forcing such as the gravitational force of another celestial body of the solar system or the Sun. These responses depend on the structure and composition of the interior. In particular, the possible presence of liquid layers inside a body and the elastic or inelastic properties of the solid parts drive the object's response to the external forcing. For example, when there are liquid layers within a body, such as Mercury or the icy satellites, the librational response of the solid part is amplified. A similar effect is expected for the nutation when considering rapid orientation changes in for example Earth and Mars (resonance to the Free Core Nutation). The rotational behavior of Ceres is thus determined by its composition and physical properties. Hence the observation of the response of Ceres to any forcing may provide information about its interior.

The purpose of this paper is to investigate the temporal variations in Ceres' rotation in order to identify potential observations to be performed by the Dawn mission. To date, observations of Ceres' surface have been obtained with the Hubble Space Telescope (HST), adaptive optics (Keck Telescope), and occultations. However, it has proven difficult to identify surface landmarks with enough accuracy to constrain the polar orientation and rotation of the asteroid (Thomas et al. 2005; Carry et al. 2008; Drummond \& Christou 2008). Owing to the nonspherical shape of Ceres related to the centrifugal potential, the gravitational potential of the Sun exerts a non-zero torque on Ceres' dynamical figure. Consequently, Ceres' axis of figure is 
A\&A 535, A43 (2011)

Table 1. Range of main parameters used in the models tested for this study for the stratified model (Fig. 1).

\begin{tabular}{|c|c|c|c|c|}
\hline Layer & $\begin{array}{r}\text { Thickness } \\
(\mathrm{km})\end{array}$ & $\begin{array}{l}\text { Density } \\
\left(\mathrm{kg} / \mathrm{m}^{3}\right)\end{array}$ & $\begin{array}{r}\text { Viscosity } \\
\eta(\mathrm{Pa} \mathrm{s}) \\
\end{array}$ & $\begin{array}{r}\text { Shear modulus } \\
\mu(\mathrm{GPa}) \\
\end{array}$ \\
\hline Dry Silicate Core & $0-275$ & 3300 & $10^{21}$ & 30 \\
\hline Hydrated Silicate Core & $125-414$ & 2700 & $10^{20}$ & 30 \\
\hline Outer Icy Shell & $25-70$ & 931 & $10^{12}-10^{19}$ & 3.3 \\
\hline
\end{tabular}

Notes. Ceres' mean radius and density are $476.2 \mathrm{~km}$ and $2078 \mathrm{~kg} / \mathrm{m}^{3}$, respectively.

expected to exhibit a precessional motion around its normal to the orbital plane and the periodic part of the torque generates a nutational motion along the precessional cone. In addition, in the reference frame of Ceres, the spin axis describes a polar motion or wobble around the figure axis, and, finally, tidal deformations arising from the Sun induce perturbations in its rotational velocity, leading to length-of-day (l.o.d.) variations.

The first part of this article describes the shape and interior structure parameters used to model Ceres. We then review the observed pole position and briefly discuss its secular evolution. In Sect. 4, we introduce the main equations used to determine the rotational parameters of the body. The geophysical and rotational models are then combined to compute the rotational motion of Ceres as a function of polar motion and precessionnutation (Sect. 5). On the basis of these results, we discuss the prospects for characterizing Ceres' rotation with the Dawn mission, and the constraints these observations will provide on Ceres' interior (Sect. 6).

\section{The shape and interior structure of Ceres}

Available constraints on Ceres' interior come from ground-based and space telescope observations and best estimates of the density and mean radius are gathered and discussed in McCord \& Sotin (2005) and Castillo-Rogez \& McCord (2010) (Table 1). Key information about the interior structure comes from shape data, which led to constraints on the mean moment of inertia, assuming that the object is in hydrostatic equilibrium. Evidence of Ceres' shape hydrostaticity was first suggested by Millis et al. (1987) based on 13 ground-based occultation observations, who concluded that the asteroid is an oblate spheroid. This configuration was confirmed by Thomas et al. (2005) from a dataset of 380 images obtained with the HST over $80 \%$ of Ceres' rotation period, as well as from other observing campaigns (e.g., Carry et al. 2008; Drummond \& Christou 2008). While it is impossible to rule out the possibility that Ceres bears non-hydrostatic anomalies with amplitudes on the order of the current uncertainty in the shape data $(\sim 2 \mathrm{~km})$, we assume as a working hypothesis that the asteroid is in hydrostatic equilibrium. We discuss the validity of this assumption in Sect. 6.

Several shape models have been suggested in the past decade, inferred from different measurement techniques. Although the data are globally consistent, they are in slight disagreement, as summarized in Zolotov (2009) and CastilloRogez \& McCord (2010). This difference is in part caused by the difference in the surface coverage enabled by the various techniques. As pointed out by Rivkin \& Volquardsen (2009), longitudinal variations in the surface composition are likely to induce a bias in the interpretation of optical images obtained over a short longitudinal range. The difference between the $(a-c)$ radii differences (where $(a)$ and $(c)$ are the equatorial and polar radii, respectively) inferred by Thomas et al. (2005) and Carry et al. (2008) is significant, on the order of $8 \mathrm{~km}$, i.e., beyond the error bars of $2 \mathrm{~km}$ estimated in both cases. The difference between the equatorial $(a)$ and the polar $(c)$ radii varies from 31.5 to $35.5 \mathrm{~km}$. The upper bound suggests the absence of large density gradients across Ceres' interior. Zolotov (2009) inferred from that observation that Ceres is not chemically differentiated and that a small density gradient is due to the variation in porosity with depth, while Castillo-Rogez \& McCord (2010) showed that even a warm icy satellite model whose core is dominated by hydrated silicate is consistent with that upper bound. Castillo-Rogez (2011) demonstrated that hydrated minerals dehydrate in response to the moderate temperature increase undergone by Ceres in the course of its evolution. A smaller value of $(a-c)$ is the signature of an increasing concentration with depth, for example caused by an inner core composed of dry silicates (ordinary chondrite-like composition) that has not evolved since accretion or result from the dehydration of hydrated silicates (Castillo-Rogez \& McCord 2010). To compute the rigid and non-rigid responses of Ceres to external perturbations, we assume that the asteroid is stratified in a rocky and icy shell, after Castillo-Rogez \& McCord (2010). The main characteristics of this interior model are summarized in Fig. 1, and the geophysical parameters tested in this study are presented in Table 1.

The mean moment of inertia is computed from the density profile:

$I=\frac{8 \pi}{3} \int_{V_{\text {body }}} \rho(r) r^{4} \mathrm{~d} r$,

where $V_{\text {body }}$ is the volume of the body, and $\rho(r)$ is the density inside the body as a function of radius $r$. The geophysical information contained in the global shape and degree-two gravity coefficients is contained in the secular tidal Love numbers $k_{\mathrm{s}}$ (Munk \& MacDonald 1960, and defined in Eq. (23))

$J_{2}=\frac{1}{3} m k_{\mathrm{s}}\left(\frac{R}{a}\right)^{2}$,

where $m$ is the rotational parameter equal to $\Omega^{2} R^{3} / G M, \Omega$ is the angular rotation rate, $\mathcal{G}$ the gravitational constant, $M$ the asteroid mass, and $R$ is mean radius. To relate the internal structure to the observables, we can used the Radau-Darwin relationship written, in its approximated form, as (Van Hoolst et al. 2008)

$$
\frac{I}{M R^{2}}=\frac{2}{3}\left(1-\frac{2}{5} \sqrt{\frac{4-k_{\mathrm{s}}}{4+k_{\mathrm{s}}}}\right),
$$

where the body is assumed to be in hydrostatic equilibrium. We can then calculate the values of the equatorial and polar moments of inertia $A$ and $C$ from:

$$
\begin{aligned}
\frac{C}{M R^{2}} & =\frac{I}{M R^{2}}+\frac{2}{3} J_{2}, \\
\frac{A}{M R^{2}} & =-J_{2}+\frac{C}{M R^{2}},
\end{aligned}
$$




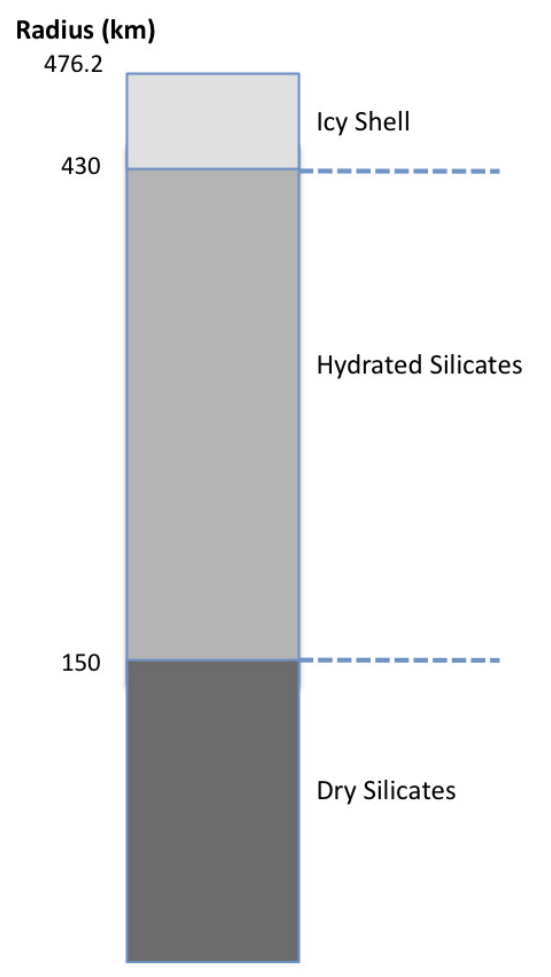

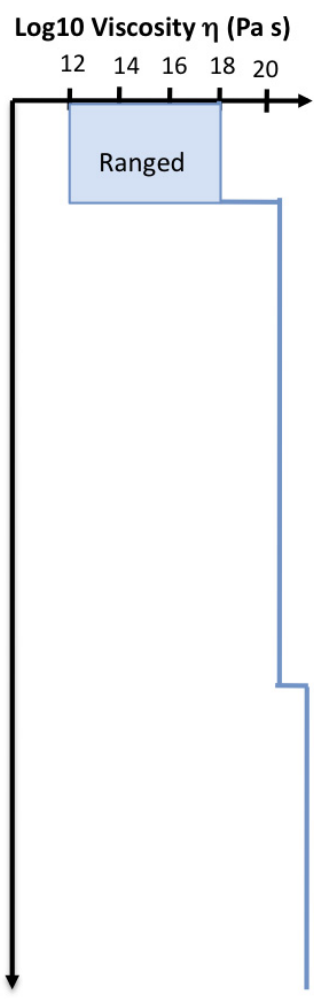

Fig. 1. Interior model of Ceres used in this study. The panels show, from left to right, the petrological structure, the corresponding density profile, and the viscosities assumed in the different layers. where $J_{2}$ is the degree-two gravity coefficient. For the model presented in Fig. 1, we obtain a mean moment of inertia $I / M R^{2}$ equal to $0.347, A / M R^{2}=B / M R^{2}=0.3394$, and $C / M R^{2}=$ 0.3623 .

We compute the complex tidal Love number $k_{2}$, from the integration of the equations of motion by, e.g., Takeuchi \& Saito (1972) (see Tobie et al. 2005; and Castillo-Rogez et al. 2011, for details about the computational approach). Ceres' dissipation factor is inferred from the imaginary part of $k_{2}$. Mechanical attenuation is computed after the composite dissipation law introduced by Castillo-Rogez et al. (2011). That model is based on the observation that the attenuation spectrum of planetary materials shows a major shift in frequency-dependence as a function of the Maxwell time $\tau_{M}$ characterizing these materials (ratio of the viscosity to the shear modulus). At forcing frequencies greater than $2 \pi / \tau_{\mathrm{M}}$, the dependence of the dissipation factor $Q$ on the angular frequency $\chi$ is such that $Q^{-1} \sim \chi^{-\gamma}$ with $\gamma=0.2-0.4$. At low frequencies, the dissipation factor follows a Maxwellian behavior such that $\gamma=1$. This change reflects an evolution in the microstructural mechanisms driving dissipation which are anelasticity-driven at high frequency and viscositydriven at low frequency ${ }^{1}$. Castillo-Rogez et al. (2011) parameterized anelasticity using the Andrade model, with application to Iapetus, an icy satellite subject to a tidal stressing of a few $\mathrm{kPa}$. The tidal stress amplitude in Ceres is on the order of $100 \mathrm{~Pa}$, thus a priori we expect the response to that stress to involve the same physical mechanisms described by Castillo-Rogez et al. (2011) for Iapetus. For the parameters displayed in Table 1, we find that $k_{2}$ is on the order of $10^{-3}$. Considering the absence of robust constraints on Ceres' temperature profile, a detailed calculation of the dissipation factor is meaningless. However, that

\footnotetext{
1 Anelastic strain is recoverable, but is a source of internal friction as it involves the motion of lattice defects. Viscoelastic strain involves the same defects, but is not recoverable.The anelasticity of planetary materials has been well-studied, and a review can be found in McCarthy \& Castillo-Rogez (2011).
}

parameter can be roughly quantified as a function of frequency. Castillo-Rogez et al. (2011) demonstrated that the dissipation factor of a water-rich object tends toward 1 at Ceres' orbital period (1681 days), but can be significantly greater than 100 at forcing periods as short as Ceres' spin period.

\section{Ceres' pole position}

\subsection{Polar orientation}

The precession-nutation theory of Ceres is defined for a given pole position of Ceres in space. However, the determination of the pole position of Ceres is difficult because of the size of the object and the lack of outstanding spectral features on its surface. In this first section, we use the available observations based on adaptive optics and HST (Thomas et al. 2005; Drummond \& Christou 2008; Carry et al. 2008) to address Ceres' pole position. The orientation data for Ceres' pole are provided in the International Celestial Reference Frame (ICRF) and are listed in Table 2. The first column represents the right ascension $\alpha_{\mathrm{s}}$, the second column the declination $\delta_{\mathrm{s}}$, and the last column the error bars. Figure 2 displays the three spin pole determinations of Table 2 projected onto the $X Y$ plane of the ICRF, i.e. $X_{\mathrm{ICRF}}$ and $Y_{\text {ICRF }}$

$$
X_{\mathrm{ICRF}}=\cos \delta \cos \alpha,
$$$$
Y_{\mathrm{ICRF}}=\cos \delta \sin \alpha \text {. }
$$

The three determinations overlap and their intersection is centered on $\alpha_{\mathrm{s}}=289.658 \mathrm{deg}, \delta_{\mathrm{s}}=63.189 \mathrm{deg}$, i.e. $\left(X_{\mathrm{ICRF}}=\right.$ $\left.0.1517, Y_{\mathrm{ICRF}}=-0.4248\right)$, indicated by a cross in Fig. 2 .

\subsection{Obliquity}

Figure 2 shows the position of the orbital pole (black point) computed from the Horizons ephemerides (Giorgini et al. 1996) and 
Table 2. Polar orientation of Ceres in right ascension $\left(\alpha_{\mathrm{s}}\right)$, declination $\left(\delta_{\mathrm{s}}\right)$, and uncertainties on each parameter $(\Delta)$.

\begin{tabular}{lrrr}
\hline \hline References & $\alpha_{\mathrm{s}}(\mathrm{deg})$ & $\delta_{\mathrm{s}}(\mathrm{deg})$ & $\Delta(\mathrm{deg})$ \\
\hline Thomas et al. (2005) & 291 & 59 & 5 \\
Drummond \& Christou (2008) & 293 & 63 & 4 \\
Carry et al. (2008) & 288 & 66 & 5
\end{tabular}

\begin{tabular}{lrr}
\multicolumn{3}{c}{ Orbital orientation } \\
& $\alpha_{n}(\mathrm{deg})$ & $\delta_{n}(\mathrm{deg})$ \\
\hline Horizons (Giorgini et al. 1996) & 293.39 & 62.85 \\
\hline
\end{tabular}

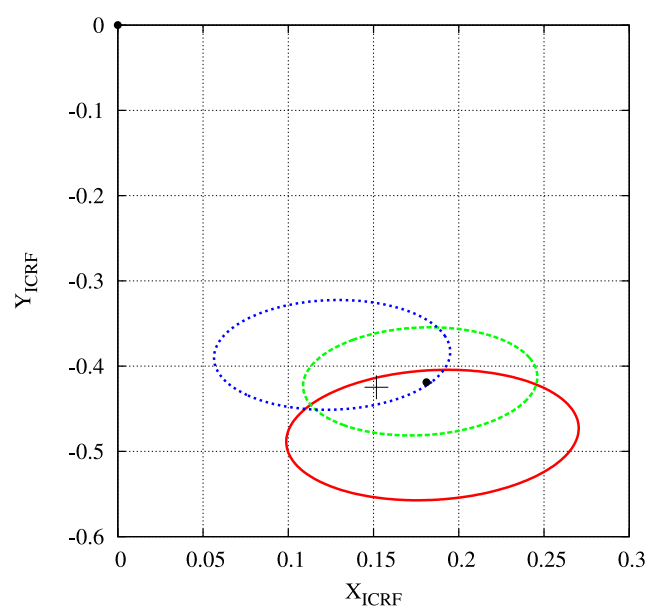

Fig. 2. Projection of the positions of the pole of rotation in the ICRF plane inferred by Thomas et al. (2005), red line; Carry et al. (2008), blue dotted line; Drummond \& Christou (2008), green shaded line, from space telescope and ground-based measurements. The black dot represents the position of the orbital pole (Giorgini et al. 1996). The cross represents the mean intersection of the three measurements and the ellipses the uncertainties.

listed as the last line of Table 2. The orbital pole location coincides with the mean value of the pole position from Drummond et al. (2008). It is then interesting to investigate the information contained in the obliquity value, as previously suggested by Bills \& Nimmo (2010). The obliquity $\varepsilon$ is defined as the angle between the normal to the orbital plane and the figure axis of Ceres. If Ceres' obliquity has reached its equilibrium position as a consequence of internal dissipation, it is possible to obtain a relationship between the obliquity and the moment of inertia known as a Cassini state (e.g. Yoder 1995)

$\frac{v}{n} \sin (\varepsilon-I)=-\frac{3}{4} \sin 2 \varepsilon \frac{C-A}{C}$,

where $v=-50.48 \mathrm{kyr}$ and $I=10.6 \mathrm{deg}$ are the precession period and inclination of the orbit of Ceres with respect to the ecliptic plane (which is taken to be coincident with the Laplace plane), $n$ is the mean motion, and $A, C$ are the moments of inertia of Ceres (here, $A=B<C$ ). This formulae comes from the generalized Cassini states that result from an equilibrium position of the spin axis by taking into account the precessing orbit of the body (Colombo 1966; Peale 1969; Henrard \& Murigande 1987; Lemaitre et al. 2006). By using a simple uniform precessional orbital period at $50.48 \mathrm{ky}$ (Bills \& Nimmo 2010) and our model of Ceres differentiated into a rocky core and icy shell (Sect. 2), we obtain an equilibrium obliquity of $\sim 0.01 \mathrm{deg}$. This small value is mainly due to the long precession period ( $\sim 50.48 \mathrm{kyr})$ with respect to the orbital period (1681 days). The secular motion of

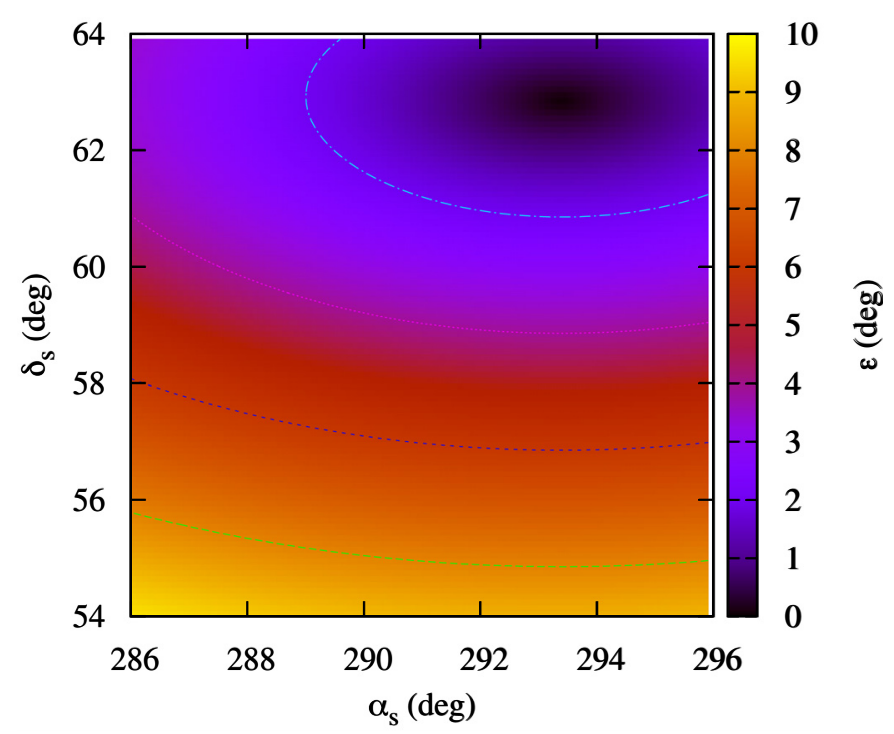

Fig. 3. Obliquity of Ceres as a function of the orientation of its spin orientation in the equatorial reference frame. The nominal value detected by Thomas et al. (2005) is $\alpha_{\mathrm{s}}=291 \pm 5 \mathrm{deg}$ and $\beta_{\mathrm{s}}=59 \pm 5 \mathrm{deg}$. The curves represent isocontours every 2 degrees.

Ceres is influenced by the oscillation at $-22 \mathrm{kyr}$ (see Bills \& Nimmo 2010) and in this case the equilibrium obliquity is equal to $0.02 \mathrm{deg}$. Relaxation to the Cassini state is achieved when the obliquity $\varepsilon$ meets this equilibrium criterion.

From the right ascension $\alpha_{n}$ and declination $\delta_{n}$ of the orbit pole, we could express the obliquity as

$\cos \varepsilon=\sin \delta_{\mathrm{s}} \sin \delta_{n}+\cos \delta_{\mathrm{s}} \cos \delta_{n} \cos \left(\alpha_{\mathrm{s}}-\alpha_{n}\right)$.

To compute the orbit pole coordinates, we use the Horizons ephemeris that provides the orbital coordinates in the ecliptic reference frame (the orbital inclination of Ceres and the ascending node are $10.6 \mathrm{deg}$ and $80.5 \mathrm{deg}$, respectively). We then express these coordinates in the ICRF by using the Earth's obliquity. The final coordinates of the orbital pole are reported in Table 2. The obliquity is equal to 4.01 degrees for the mean pole orientation of Thomas et al. (2005), $0.23 \mathrm{deg}$ for Drummond \& Christou (2008), and $3.91 \mathrm{deg}$ for Carry et al. (2008). The observation of Drummond \& Christou (2008) seems to be close to that expected if Ceres is relaxed to a Cassini state. The uncertainty in the obliquity is represented in Fig. 3 in the case of the measurement obtained by Thomas et al. (2005). The obliquity is between 0 and 10 degrees i.e. that contains the Cassini state but displays a very large uncertainty. For this paper, we use as a working reference for Ceres' orientation the upper value of the obliquity of $9.6 \mathrm{deg}$. The reason for using this large value, while data overlap for an obliquity value of $\sim 3 \mathrm{deg}$, is that it will yield upper bounds on our estimates of the rotational perturbations. This will help us to assess whether or not these perturbations can be measured with spaceborne techniques.

Bills \& Nimmo (2010) predicted that the obliquity of Ceres is $\sim 12$ deg based on a secular orbital model of Ceres. However, available observations (e.g. Thomas et al. 2005) and the location of the orbital pole yielded by the Horizons ephemeris indicate that the obliquity is most likely between 0 and $10 \mathrm{deg}$. The discrepancy seems to reside in the initial value used in Eq. (20) of Bills \& Nimmo (2010) and could be resolved by using the initial values output by the Horizons ephemerides. 


\subsection{Damped obliquity}

We now estimate the timescale for Ceres to evolve toward that equilibrium state. The obliquity damping rate $\dot{\varepsilon}$ may be computed from equations (e.g., Néron de Surgy et al. 1996; Levrard et al. 2007) describing the secular rotational evolution of Ceres (for an orbit without planetary perturbations) given by

$$
\begin{aligned}
\dot{\varepsilon} & =\frac{K n}{C \Omega} \sin \varepsilon\left[\cos \varepsilon f_{1}(e) \frac{\Omega}{2 n}-f_{2}(e)\right], \\
\dot{\Omega} & =-\frac{K n}{C}\left[f_{1}(e) \frac{1+\cos \varepsilon^{2}}{2} \frac{\Omega}{n}-f_{2}(e) \cos \epsilon\right]
\end{aligned}
$$

where

$$
\begin{aligned}
& f_{1}(e)=\frac{1+3 \mathrm{e}^{2}+3 \mathrm{e}^{4} / 8}{\left(1-\mathrm{e}^{2}\right)^{9 / 2}} \\
& f_{2}(e)=\frac{1+15 \mathrm{e}^{2} / 2+45 \mathrm{e}^{4} / 8+5 \mathrm{e}^{6} / 16}{\left(1-\mathrm{e}^{2}\right)^{6}}
\end{aligned}
$$

$e$ is the eccentricity, and the constant $K$ is defined as

$K=3 \frac{k_{2}}{Q} \frac{G M^{2}}{R_{\mathrm{e}}}\left(\frac{M_{\odot}}{M}\right)^{2}\left(\frac{R_{\mathrm{e}}}{D}\right)^{6} n$,

where $\mathcal{G}$ is the gravitational constant, $M_{\odot}$ the mass of the Sun, $D$ Ceres' semi-major axis, $R_{\mathrm{e}}$ its equatorial radius, and $\Omega$ the angular rotation rate. The parameters $k_{2}$ and $Q$ correspond to the tidal Love number and dissipation factor, respectively, at the orbital frequency. For the sake of simplicity, we assume for this calculation that these parameters remain constant. The inverse dependence of that equation on $D^{6}$ indicates that Ceres' large distance to the Sun is a severe limitation to any tidal evolution of its dynamical properties. In addition, we neglect in this expression the impact of orbital perturbations.

From the system of equations in Eq. (11), as quoted by Correia (2009) the timescale of evolution of the rotation rate is shorter than the timescale for the obliquity evolution. Hence it is expected that the rotation first reaches its equilibrium that will take a damping rate on the order of

$\dot{\Omega} \sim 6.14^{-11} \frac{k_{2}}{Q}, \quad \dot{\varepsilon} \sim 1.00^{-14} \frac{k_{2}}{Q}$,

where time is expressed in years. The parameters $k_{2}$ and $Q$ are unknown but may be approached from geophysical modeling. We note that Bills \& Nimmo (2010) considered a situation where Ceres' material is in equilibrium at the dissipation peak. However, this situation is less than likely. The contribution of tidal dissipation to the total heat budget of the object is negligible (below $0.1 \%$ ) in comparison to insolation and, to a lesser extent, long-lived radioisotopes. This precludes the ability of that heat source to drive the geophysical state of the asteroid. For the reference model chosen for this study (detailed in the previous section), differentiated in an icy shell and rocky core, $k_{2}$ is on the order of $10^{-3}$ and $Q$ is on the order of 10 . In that case, the dissipation time is on the order of $10^{13}$ years for $\dot{\Omega}$ and $10^{17}$ years for $\dot{\varepsilon}$. Hence, we infer that Ceres' obliquity has probably not been fully damped over its lifetime. As a consequence, our result contrasts with the conclusion of Bills \& Nimmo (2010) that Ceres' obliquity could have been damped in a few hundred Myr, even if we assume that the asteroid is in a very dissipative state.

\section{Rotational model of Ceres}

\subsection{Euler-Liouville equations}

If Ceres were perfectly spherical and rigid, then its rotation would be uniform. However, the HST measurements have shown that the figure of Ceres is an oblate body (within the error bars), from which we inferred an equatorial oblateness $\alpha=(C-A) / A=$ 0.0675 (see Sect. 2). Thus, the Sun exerts a non-zero torque on Ceres dynamical figure, which responds in the form of the precession and nutation of its orientation axes. The Sun also raises tides that deform its surface and perturb its rotational velocity.

Thus, it is convenient to describe Ceres' rotation using the approach developed for Earth, which is oblate to first order. The rotation of the body is described through the classical Euler-Liouville equation written as (see Moritz \& Mueller 1987; Dehant \& Mathews 2007)

$\frac{\mathrm{d} \boldsymbol{H}}{\mathrm{d} t}+\boldsymbol{\Omega} \wedge \boldsymbol{H}=\boldsymbol{\Gamma}$.

This describes the variations in the angular momentum $\boldsymbol{H}$ disturbed by an external torque $\boldsymbol{\Gamma}$. This equation is expressed in the rotating frame tied to the body through the spin velocity $\boldsymbol{\Omega}$ and written in the Tisserand frame (Munk \& MacDonald 1960). As shown in Sect. 6, the wobble damping time is some ten thousands of years and we could assume that the instantaneous axis of rotation is near the polar principal axis of the body. Thus,

$\boldsymbol{\Omega}=\left(\begin{array}{c}m_{1} \\ m_{2} \\ 1+m_{3}\end{array}\right) \Omega$,

where $\Omega$ is the mean rotation of the body and the quantities $m_{i}$ are small and dimensionless. The pair $m_{1}, m_{2}$ describes the polar motion of Ceres, i.e. the orientation of the rotational speed in the body reference frame, while $m_{3}$ corresponds to the variation in the rotational speed as shown in the linearized expression

$\|\boldsymbol{\Omega}\|=\Omega\left(1+m_{3}\right)$.

The angular momentum is $\boldsymbol{H}=I \boldsymbol{\Omega}$ where $I$ is the tensor of inertia of the body expressed as

$I=\left(\begin{array}{ccc}A & 0 & 0 \\ 0 & A & 0 \\ 0 & 0 & C\end{array}\right)+\left(\begin{array}{lll}c_{11} & c_{12} & c_{13} \\ c_{21} & c_{22} & c_{23} \\ c_{31} & c_{32} & c_{33}\end{array}\right)$,

where the $c_{i j}$ are symmetric and represent the departure from the reference ellipsoid, i.e. the deformation of the body surface. By introducing the moment of inertia Eq. (18) into the dynamical equations Eq. (15) and developing at first order in $m_{j}$ and $c_{i j}$, the linearized dynamical equations are

$$
\begin{aligned}
A \Omega \dot{m}_{1}+(C-A) \Omega^{2} m_{2}+\Omega \dot{c}_{13}-\Omega^{2} c_{23} & =L_{1} \\
A \Omega \dot{m}_{2}-(C-A) \Omega^{2} m_{1}+\Omega \dot{c}_{23}+\Omega^{2} c_{13} & =L_{2} \\
C \Omega \dot{m}_{3}+\Omega \dot{c}_{33} & =L_{3}
\end{aligned}
$$

or by introducing complex notations, as usual for Earth rotation studies, $m=m_{1}+\mathrm{i} m_{2}, L=L_{1}+\mathrm{i} L_{2}$, and $c=c_{13}+\mathrm{i} c_{23}$, we obtain one complex equation for the polar motion

$A \dot{m}-\mathrm{i} \alpha A \Omega m+\dot{c}+\mathrm{i} \Omega c=\frac{L}{\Omega}$

and one equation for the 1.o.d. variations

$C \Omega \dot{m}_{3}+\Omega \dot{c}_{33}=L_{3}$.

We note that, for the approximation of an axisymmetric body, the polar motion and 1.o.d. variation are described by independent dynamical equations. 


\subsection{Deformed tensor of inertia}

The centrifugal and tidal potentials deform the body and that deformation may be expressed as (Dehant et al. 2005)

$c=\alpha A \frac{k_{2}}{k_{\mathrm{s}}} m-3 \alpha A \frac{k_{2}}{k_{\mathrm{s}}} \frac{W_{21}}{\Omega^{2} d^{2}}$,

where the first term results from the centrifugal potential and the second one from the tidal potential and especially the tesseral potential $W_{21}$ (see Sect. 4.4), $\mathcal{G}$ is the gravitational constant, $d$ is the mean Sun-Ceres distance, $k_{2}$ is the tidal Love number, and $k_{\mathrm{S}}$ the secular Love number defined as (Munk \& MacDonald 1960)

$k_{\mathrm{s}}=3 \frac{(C-A) \mathcal{G}}{\Omega^{2} R^{5}}$,

where $R$ the radius of the surface.

The $c_{33}$ tensor varies as a function of the centrifugal and tidal potentials after (Greff-Lefftz et al. 2000)

$c_{33}=-\frac{4}{3} \alpha A \frac{k_{2}}{k_{\mathrm{s}}} m_{3}-2 \alpha C \frac{k_{2}}{k_{\mathrm{s}}} \frac{W_{20}}{\Omega^{2} d^{2}}$,

where $W_{20}$ is the zonal potential (see Sect. 4.4).

\subsection{Gravitational torque}

The tesseral degree-two tidal potential $W$ acts on the Ceres' equatorial bulge. Thus involves an equatorial torque such that (Dehant \& Mathews 2007)

$L_{1}+\mathrm{i} L_{2}=-\frac{3 \mathrm{i} \alpha A}{d^{2}} W_{21}$,

where the complex potential $W_{21}$ is developed in the next section. The torque $L_{3}$ is equal to zero because of the symmetry axis of Ceres.

\subsection{The tidal potential}

The gravitational tidal potential induced by the Sun may be expressed in both a frame tied to Ceres $(\mathrm{MBRF}=$ mean body reference frame) and the celestial frame $(\mathrm{MCRF}=$ mean celestial reference frame). Choosing one reference frame, phenomena induced by the gravitational forcing have to be expressed in the same frame, with the particularities that the frequency in the frame tied to Ceres and the frequency in space are related by the rotational velocity of the body (see Eq. (29)). Hence a constant torque applied in the MCRF will appear to be periodic in the MBRF at the rotational frequency and vice versa.

Following the method of Dehant \& Mathews (2007), the degree-two potential exerted by the Sun on Ceres in the MBRF is developed as $W_{2}=W_{20}+W_{21}$ where

$$
W_{20}=\frac{G M}{d^{3}}\left(z^{2}-\frac{1}{3}\right)
$$

and

$$
W_{21}=\frac{\mathcal{G M}}{d^{3}}(x z+\mathrm{i} y z)
$$

where the $W_{20}$ leads to the zonal part and $W_{21}$ leads to the sectorial part of the tidal torque. Here, the sectorial part is zero because of the axi-symmetric of the body's shape. The cosine directions $(x, y, z)$ are the direction of the Sun in the MBRF. They are evaluated from the Horizons ephemeris (Giorgini et al. 1996) and rotated from the ecliptic frame to the MBRF by using the polar direction of Ceres with a right ascension of $286 \mathrm{deg}$ and declination of $54 \mathrm{deg}$ consistent with an obliquity of $9.2 \mathrm{deg}$. The uncertainty related to the direction of the polar direction of Ceres is discussed in Sect. 3.1.

The zonal part is developed as a Fourier series $W_{20}=$ $W_{20}^{j} \mathrm{e}^{\mathrm{i} \omega_{j} t}$, where $W_{20}^{j}$ also contains the phases, and the tesseral part is developed as a Fourier series with prograde (index +) and retrograde (index -) components

$W_{21}=W_{21}^{+} \mathrm{e}^{\mathrm{i} \omega_{j} t}+W_{21}^{-} \mathrm{e}^{\mathrm{i} \omega_{-j} t}$,

where we used the same notations as Roosbeek (1995) and Roosbeek \& Dehant (1998). The prograde and retrograde circular motions allow us to express the elliptical motion in two symmetric motion components. The frequencies $\omega_{j}$ of the tidal potential are expressed in the MBRF, and they are related to the prograde and retrograde frequencies $\left(\Delta \omega_{j},-\Delta \omega_{j}\right)$ expressed in the space MCRF, by means of

$$
\begin{aligned}
\omega_{j} & =\Delta \omega_{j}-\Omega, \\
\omega_{-j} & =-\Delta \omega_{j}-\Omega,
\end{aligned}
$$

where $\Omega$ is the rotation period close to nine hours. Henceforth, the periods appear to be long in space and short in the frame tied to Ceres.

For Earth, one goes from the terrestrial reference frame tied to the planet to the celestial frame using several rotation matrices. These rotations first bring the terrestrial frame attached to the figure axis of the Earth to the intermediate pole, accounting for polar motion; a rotation is then performed along the true equator of date around this intermediate pole, accounting for the Earth's rotation (uniform part and l.o.d. or UT1 variations). The precession and nutations are then accounted to bring the true equator of date to the celestial frame. Precession and nutations are thus those of the true equator of date. One has to keep in mind however that the choice concerning the intermediate frame is purely conventional. The more logical choice is of course related to the way in which UT1 or the 1.o.d. variations are expressed. In the recently adopted conventions for Earth, the intermediate frame is the equator of the Celestial Intermediate Pole (CIP), a conventional pole that has no retrograde diurnal motion in a reference frame tied to the Earth, and only long-period motions (precession, nutations) in space. The instantaneous rotation pole and the mean rotation pole are not identical; they differ by small changes in their direction caused by the atmosphere, ocean, and hydrology excitation of polar motion on very short periods. When computing the precession and nutations, these axes have identical long-period motions in space and retrograde diurnal motion in a frame tied to the Earth. As, for Ceres, we can ignore the differences at short period in space. We then work with the instantaneous rotation pole $\left(m_{1}, m_{2}\right)$ in a frame tied to Ceres. Long-term motion in that frame will be related to the Chandler Wobble, if excited. Long-term motion of the pole in space or retrograde diurnal motions in the frame tied to Ceres represent precession and nutations. We have a one-to-one relation between the frequencies of these motions in a frame tied to Ceres and in space.

\section{Description of the rigid rotational motion}

\subsection{Polar motion}

We first solve the polar motion by introducing the potential expressed in Eq. (28) and using a Fourier transform $\mathrm{e}^{\mathrm{i} \sigma t}$ to express 
Table 3. Ceres rigid Polar motion $\left(k_{2}=0\right)$.

\begin{tabular}{lcc|ccc}
\hline \hline $\begin{array}{l}\text { Freq } \omega_{j} \\
(\mathrm{rad} / \mathrm{days})\end{array}$ & $\begin{array}{c}\text { Per } \\
\text { (days) }\end{array}$ & $\begin{array}{c}\text { Amp } \\
(\mathrm{mas})\end{array}$ & $\begin{array}{c}\text { Freq } \omega_{-j} \\
\text { (rad/days) }\end{array}$ & $\begin{array}{c}\text { Per } \\
\text { (days) }\end{array}$ & $\begin{array}{c}\text { Amp } \\
(\mathrm{mas})\end{array}$ \\
\hline-16.617 & -0.37813 & 0.1683 & & & \\
-16.613 & -0.37821 & 0.0299 & -16.620 & -0.3780 & -0.0101 \\
-16.609 & -0.37830 & 0.1638 & -16.624 & -0.3780 & 0.0011 \\
-16.605 & -0.37838 & 0.0449 & -16.628 & -0.3779 & -0.0003 \\
-16.605 & -0.37840 & 0.0009 & -16.629 & -0.3779 & 0.0000 \\
-16.602 & -0.37847 & 0.0085 & -16.632 & -0.3778 & -0.0001 \\
-16.598 & -0.37855 & 0.0014 & -16.635 & -0.3777 & 0.0000 \\
\hline
\end{tabular}

Notes. The prograde motions are shown in the left columns of the table whereas the right part of the table contains the retrograde motions.

the budget equations at a given frequency in Eq. (20). The complex polar motion is then

$$
\begin{aligned}
m= & m_{\mathrm{c}} \mathrm{e}^{\mathrm{i} \sigma_{\mathrm{c}} t} \mathrm{e}^{-\lambda t} \\
& +\sum_{j}\left[\frac{-3 \alpha W_{21}^{+}}{\Omega^{2} d^{2}} \frac{\Omega-\left(\Omega+\omega_{j}\right) k_{2} / k_{\mathrm{s}}}{\omega_{j}-\Omega \alpha+\alpha\left(\Omega+\omega_{j}\right) k_{2} / k_{\mathrm{s}}} \mathrm{e}^{\mathrm{i}\left(\omega_{j} t+\phi_{j}\right)}\right. \\
& \left.+\frac{-3 \alpha W_{21}^{-}}{\Omega^{2} d^{2}} \frac{\Omega-\left(\Omega+\omega_{-j}\right) k_{2} / k_{\mathrm{s}}}{\omega_{-j}-\Omega \alpha+\alpha\left(\Omega+\omega_{-j}\right) k_{2} / k_{\mathrm{s}}} \mathrm{e}^{\mathrm{i}\left(\omega_{-j} t+\phi_{j}\right)}\right],
\end{aligned}
$$

hence consists of a free mode (first term) and a sum of forced modes, which are prograde and retrograde.

The frequency of the free mode is called the Chandler frequency $\sigma_{\mathrm{c}}$ by analogy with Earth rotation and is written as

$\sigma_{\mathrm{c}}=\alpha \Omega \frac{k_{\mathrm{s}}^{2}-\left|k_{2}\right| k_{\mathrm{s}} \cos \delta+\left|k_{2}\right| \alpha\left(k_{\mathrm{s}} \cos \delta-\left|k_{2}\right|\right)}{k_{\mathrm{s}}^{2}+2 \alpha\left|k_{2}\right| k_{\mathrm{s}} \cos \delta+\alpha^{2}\left|k_{2}\right|^{2}}$.

Its period is about 5.48 days and the correction for the deformation $k_{2}=\left|k_{2}\right| \mathrm{e}^{(-\mathrm{i} \delta)}$, where $\delta$ is the phase lag representing the dissipative part, which is between $3 \%$ and $5 \%$ of its value. This contrast to the Earth's case, for which the deformation induces a difference of 100 days in the period. The difference in behavior comes mainly from the value of $\alpha$ that differs by about one order of magnitude between the two bodies. The amplitude $m_{\mathrm{c}}$ and the phase $\phi_{\mathrm{c}}$ of the Chandler mode depend on the dynamical and geophysical history of the body (see discussion in Sect. 6.2). The amplitude of the Chandler mode is damped on a typical timescale $1 / \lambda$, a function of the imaginary part of the Love number and expressed as

$\lambda=\frac{\alpha \Omega\left|k_{2}\right| k_{\mathrm{s}} \sin \delta(\alpha+1)}{k_{\mathrm{s}}^{2}+2 \alpha\left|k_{2}\right| k_{\mathrm{s}} \cos \delta+\alpha^{2}\left|k_{2}\right|^{2}}$.

Its value depends strongly on the interior model as discussed in Sect. 6 .

The forced terms of Ceres' rigid polar motion are shown in Table 3. The polar motion of Ceres oscillates on short periods close to nine hours and its motion projected onto the surface of Ceres is very small (see Fig. 4). Its amplitude multiplied by the mean radius of Ceres is $\sim 0.5 \mathrm{~mm}$, and summing all the contributions regardless of the phase yields an amplitude no greater than $1 \mathrm{~mm}$.

\subsection{Precession-nutation of Ceres}

The rotational motion of Ceres' polar axis describes in the inertial reference frame, the MCRF, a precessional nutational

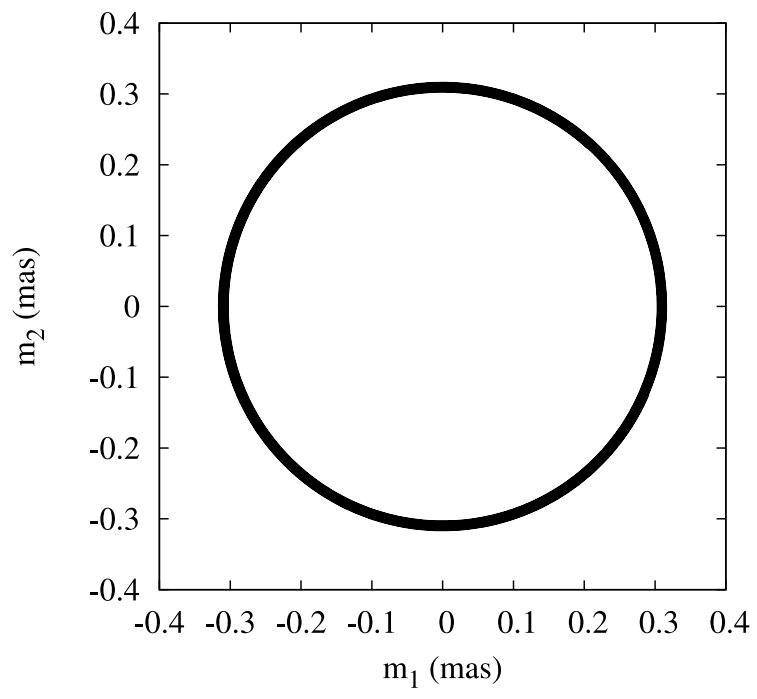

Fig. 4. The polar motion of Ceres is circular and has a main term that oscillates on 840 days.

motion. The rotation angles and their derivatives are easily computed by using the kinematic Euler equation allowing us to express the instantaneous rotation pole components in terms of the nutation angles as

$\dot{\theta}+\mathrm{i} \dot{\psi} \sin \theta=\Omega m \mathrm{e}^{\mathrm{i} \Omega t}$,

where $\Omega$ accounts for the expression of the pole in space due to the rotation around the $Z$-axis. Hence, after integrating Eq. (33), except for the case $\omega_{j}=-\Omega$ that leads to the precessional motion, we obtain the nutation series

$$
\begin{aligned}
& \Delta \theta+\mathrm{i} \Delta \psi \sin \theta=-\mathrm{i} \frac{\Omega}{\Omega+\sigma_{\mathrm{c}}} m_{\mathrm{c}} \mathrm{e}^{\mathrm{i}\left(\Omega+\sigma_{\mathrm{c}}\right) t} \mathrm{e}^{-\lambda t} \\
& +\mathrm{i} \sum_{j \neq 0}\left[\frac{-\Omega}{\Delta \omega_{j}} m_{j}^{+} \mathrm{e}^{\mathrm{i}\left(\Delta \omega_{j} t+\phi_{j}\right)}+\frac{\Omega}{\Delta \omega_{j}} m_{j}^{-} \mathrm{e}^{-\mathrm{i}\left(\Delta \omega_{j} t+\phi_{j}\right)}\right] .
\end{aligned}
$$

The rigid nutations of Ceres are described by the periodic components of the last equation and are listed in Table 4 . The amplitudes of the long-period nutations are positively affected because the amplitude is inversely proportional to the forcing frequency. The main term (in absolute amplitude) is the semi-annual nutation $2 \lambda_{\mathrm{c}}$ related to the obliquity of Ceres, and then the terms related to harmonics. We also note the presence of a term related to Jupiter's mean longitude $\lambda_{\mathrm{J}}$. The amplitude of the annual nutation is around 364 mas, which represents a $0.84 \mathrm{~m}$ surface displacement, for a mean radius of $476 \mathrm{~km}$. The detection of this small displacement requires tracking of Ceres' surface with a beacon for an extensive period of time.

The first term in Eq. (34) represents the Chandler mode observed from space. In this case, it has a period of $9 \mathrm{~h} 40 \mathrm{~min}$ i.e. an increase of $36 \mathrm{~min}$ with respect to the proper rotation of the body. The precessional motion of the figure axis is represented by the oscillation at $\omega_{j}=-\Omega$ in the body reference frame that is purely imaginary $m_{0}=0.1576$ mas, leading to a precession time of 226981.8 years that is longer than the 218654.2 year period calculated with the classical formulae

$\dot{\psi}=-\frac{3}{2} \frac{n^{2}}{\Omega} \frac{C-A}{C} \cos \epsilon$

The discrepancy between the two results (4\%) is essentially due to the ephemeris length used in the frequency analysis of the potential. 
Table 4. Ceres rigid nutations $\left(k_{2}=0\right)$ and corresponding argument with $\lambda_{\mathrm{c}}$ and $\lambda_{\mathrm{J}}$ mean longitudes of Ceres and Jupiter.

\begin{tabular}{lcccc}
\hline \hline Arg & $\begin{array}{c}\text { Freq } \\
\text { (rad/days) }\end{array}$ & $\begin{array}{c}\text { Per } \\
\text { (days) }\end{array}$ & $\begin{array}{c}\text { Amp Nut prog } \\
\text { (mas) }\end{array}$ & $\begin{array}{c}\text { Amp Nut retro } \\
\text { (mas) }\end{array}$ \\
\hline $2 \lambda_{\mathrm{c}}$ & 0.00747 & 840.8 & -364.296 & 2.504 \\
$\lambda_{\mathrm{c}}$ & 0.00374 & 1681.7 & -133.129 & -44.976 \\
$3 \lambda_{\mathrm{c}}$ & 0.01121 & 560.6 & -66.627 & -0.400 \\
$4 \lambda_{\mathrm{c}}$ & 0.01495 & 420.4 & -9.449 & -0.054 \\
$4 \lambda_{\mathrm{c}}-\lambda_{\mathrm{J}}$ & 0.01204 & 521.7 & -1.256 & 0.009 \\
$5 \lambda_{\mathrm{c}}$ & 0.01868 & 336.3 & -1.217 & 0.007 \\
\hline
\end{tabular}

Notes. The amplitudes are truncated at $10^{-4}$ mas.

Table 5. $m_{3}$ variations of Ceres for a tidal Love number $k_{2}=$ $0.017-\mathrm{i} 1.27 \times 10^{-7}$ with frequencies expressed in the inertial reference frame.

\begin{tabular}{lccc}
\hline \hline $\begin{array}{l}\text { Freq } \\
\text { (rad/days) }\end{array}$ & $\begin{array}{c}\text { Per } \\
\text { (days) }\end{array}$ & $\begin{array}{c}\text { Amp in-phase } \\
\text { (mas) }\end{array}$ & $\begin{array}{c}\text { Amp out-of-phase } \\
\text { (mas) }\end{array}$ \\
\hline 0.00374 & 1681.9 & 0.00072 & 0.0 \\
0.00747 & 840.8 & 0.00020 & 0.0 \\
0.01121 & 560.6 & 0.00004 & 0.0 \\
0.00457 & 1374.3 & 0.00001 & 0.0 \\
\hline
\end{tabular}

Notes. The amplitudes are truncated at $10^{-5}$ mas.

\section{Geophysical constraints from space observations}

\subsection{Non-rigid contributions: I.o.d.}

In Sect. 4, we introduced the rotational equations for a non-rigid body. The application of these equations to the geophysical models shows that the non-rigid contributions to the shape deformation bear a negligible effect on both polar motion and nutations. However, the variations in the moments of inertia in response to the tidal forcing exerted on the body generate a non-zero torque along the figure axis that would perturb the uniform rotational motion in the form of 1.o.d. variations. By combining Eq. (21) and the inertia deformation in Eq. (24), we deduce the variations in the 1.o.d. $m_{3}$

$$
m_{3}=\frac{2 \alpha \frac{k_{2}}{k_{\mathrm{s}}}}{1-\frac{4}{3} \alpha \frac{A}{C} \frac{k_{2}}{k_{\mathrm{s}}}} \frac{W_{20}^{j}}{\Omega^{2} d^{2}} .
$$

The resulting oscillations in the $m_{3}$ variations are under 0.001 mas (Table 5), largely below the expected accuracy for space-borne observational techniques. The term at 1374.3 days is related to Jupiter with the combination $2 \lambda_{\mathrm{c}}-\lambda_{\mathrm{J}}$.

\subsection{Wobble}

The rotational motion of Ceres appears to be relatively uniform because all the nutational oscillations, polar motion, and l.o.d. variations have very small amplitudes. Therefore, if a sizeable departure from a quiet rotation is detected by the Dawn mission at a period of about $9 \mathrm{~h} 40$, then we could assign this motion to the Wobble. The presence of a Chandler mode is indeed expected as soon as any perturbation, exterior or interior to the body, shifts the figure axis from its equilibrium position. However, this mode is also damped because of the internal dissipation. The Chandler mode expressed in the inertial reference frame has a period of $9 \mathrm{~h} 40 \mathrm{~min}$, i.e., around $36 \mathrm{~min}$ longer than the proper rotation of the body. As the nutations in the inertial frame have long periods

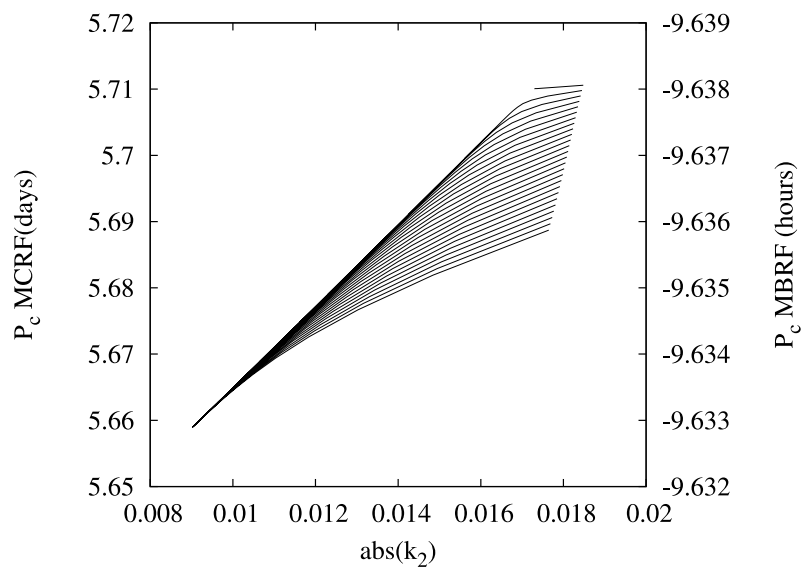

Fig. 5. Period of the Chandler model as a function of the value of the Love number $k_{2}$. The lower bound of $k_{2}$ corresponds to a fully frozen model, while the upper bound is expected if Ceres contains a global ocean.

(harmonics of the orbital period), then any observed departure of the uniform rotation at the short period of $9 \mathrm{~h} 40$ might be attributed to the Chandler mode. The Chandler period is sensitive to the value of the Love number $k_{2}$ as shown in Fig. 5, where the period is expressed in both the reference frame MBRF (5.5 days) and MCRF (9h40). We consider a wide range of possible values for $k_{2}$ covering the spectrum of possible models envisioned for Ceres. This parameter is computed at the period of the Chandler mode of 5.5 days, i.e. the period in the body reference frame. The damping timescale $T_{\lambda}$ is proportional to $Q$ and the damping timescale can be as long as 120000 years for dissipative models with large $Q$ and as short as few decades if the object is very dissipative. Thus, the damping timescale could be very short, hence a non-zero Chandler mode requires a continuous physical process or a recent impulse to be observable today.

In the Earth's case, the Chandler wobble is mainly excited by the atmosphere and the ocean. In the case of Ceres, there is no atmosphere, but Ceres is in a rich dynamical environment, the asteroid belt, thus is exposed to a constant meteoritic flux. This meteoritic flux may involve impacts exciting the Chandler mode for Ceres. The wobble excitation may be expressed by using Peale (1975) expression introduced in the case of the Moon

$$
m_{\mathrm{e}}=-\frac{\mathrm{i} c}{A \alpha} H\left(t-t_{0}\right)+\left(-\frac{c}{A}+\frac{\mathrm{i} c}{A \alpha}+\frac{N}{A \alpha}\right) H\left(t-t_{0}\right) \mathrm{e}^{\mathrm{i} \alpha \Omega\left(t-t_{0}\right)},
$$

where $m_{\mathrm{e}}$ represents the polar response of the impact, $N$ represents the maximum angular momentum potentially induced on Ceres by a collision $(N=m v R$ with $m$ and $v$ corresponding to the mass and velocity of the bolide, respectively, and $R$ Ceres' radius). The parameter $H\left(t-t_{0}\right)$ is the Heaviside function associated with an impact at time $t_{0}$. This expression contains two components: the angular momentum transfer and the modification of the moment of inertia caused by the ejected matter and formation of a crater. The subsequent response of the pole is composed of a constant offset due to the first term in Eq. (37) and a term due to the excitation of the Chandler mode. We use the formulation of Gauchez \& Souchay (2006) for crater modeling and the scaling law is borrowed from Holsapple (1993). We search for possible impact configurations leading to the excitation of the Chandler mode with an amplitude of 10 arcsec, i.e., a displacement at the surface of $20 \mathrm{~m}$ (the amplitude observed today would be damped because of the dissipation of the 
Chandler mode, so these events have to be recent). This may be achieved for a cometary projectile (heliocentric) with a diameter of $2.5 \mathrm{~km}$, a density of $0.6 \mathrm{~g} / \mathrm{cm}^{3}$, and a velocity of $20 \mathrm{~km} \mathrm{~s}^{-1}$; or by a neighboring asteroid of $4 \mathrm{~km}$ diameter with a density of $1.3 \mathrm{~g} / \mathrm{cm}^{3}$, colliding at $5 \mathrm{~km} \mathrm{~s}^{-1}$ (Farinella \& Davis 1992).

To estimate the probability of this impact on Ceres, we survey main-belt asteroids with absolute magnitude brighter than 14. The population contains approximately 25000 objects (see Jedicke et al. 2002). Trajectories of all the considered asteroids were calculated for a 100-year time interval assuming Keplerian orbits. A fictional object evolving on the same orbit as Ceres, but with a cross-section 10000 times greater would experience 200 collisions with other asteroids. Scaling this value to Ceres' size and a time span of 150000 years, we obtain 0.003 impacts on Ceres during that timeframe. An asteroid diameter of $4 \mathrm{~km}$ corresponds approximately to an absolute magnitude of 15. According to Jedicke et al. (2002), these objects are two to three times more abundant than the population considered here. As a consequence, the corresponding number of impacts onto Ceres amounts to approximately 0.007 per 150000 yrs. The probability that Ceres experienced in the past 150000 years a collision with an object larger than $4 \mathrm{~km}$ appears to be small (less than 1\%). Such an estimate is more difficult to calculate in the case of cometary collisions owing to the lack of constraints on the possible reservoirs of comets.

Another consequence of collision with large objects is the alteration of the moments of inertia of Ceres that may lead to shift in its figure axis (first term in Eq. (37)). The long-term consequence of that effect needs to be studied in detail. This would require to properly model the respective timescales for the relaxation of the crater and the equatorial bulge. As noted by Nimmo \& Matsuyama (2007), both processes depend on the mechanical properties of the icy shell, thus should proceed on the same timeframe, which increases the complexity of the problem. For Ceres, we expect the low subsurface viscosity to promote rapid crater relaxation preventing the re-orientation.

We also checked for the possible occurrence of close encounters during the Dawn mission lifetime, which could excite Ceres' spin axis by an impulse of its gravitational torque. From realistic (non Keplerian) asteroid orbits, we found no close encounter between 2010 and 2020 that could modify the rotational dynamics of Ceres. The encounters are insufficiently close or the bodies involved are not massive enough. This estimate accounts only for main-belt asteroids with absolute magnitudes brighter than 14 .

Another source of excitation of the Chandler wobble may be an equatorial sea inside Ceres. Such a water reservoir was suggested by Castillo-Rogez \& McCord (2010) based on the observation that Ceres' surface temperature at the equator is close to the eutectic temperature of salt impurities expected in the asteroid. Several astrophysical models also suggest that Ceres accreted a significant fraction of ammonia hydrates (up to 7 wt. $\%$ of the ice phase, Dodson-Robinson et al. 2009), and possibly also methanol hydrates (Mousis et al. 2008). The presence of these compounds would help to preserve a deep liquid layer over extended periods of time, e.g. the ammonia hydrate peritectic temperature in Ceres' pressure conditions is $\sim 176 \mathrm{~K}$ (Hogenboom et al. 1997), i.e., similar to Ceres' surface temperature. The excitation process is then related to possible current circulation and loading because of the fluid reservoir. Deep liquid layer may result in increased dissipation, as suggested by Tyler (2008) in the case of outer planet icy satellites. Although the modeling of this process is beyond the scope of this paper, circulation in closed ocean systems and its signature on the rotation is an important topic in planetary sciences (e.g., Tyler 2008; Noir et al. 2009).

\subsection{Hydrostatic state}

There are multiple sources of departure from hydrostatic equilibrium on the large scale, starting with the large contrast in temperature between the equator and the poles, of at least $50 \mathrm{~K}$ (Fanale \& Salvail 1988). Castillo-Rogez \& McCord (2010) suggested that Ceres' equatorial temperature might promote the preservation of a regional deep liquid layer, while the polar regions remained entirely frozen. The contrast in density between water ice and liquid water saturated in brines can be up to $60 \%$ (e.g., Prieto-Ballesteros \& Kargel 2005), which would increase the difference between $A$ and $C$ by about $5 \%$. Another source of density anomalies are mascons (mass concentrations), for example caused by topography anomalies at the silicate core, as inferred for Ganymede from Galileo measurements (e.g., Palguta et al. 2009). Schenk \& McKinnon (2008) suggested in the case of Enceladus that an unrelaxed core is responsible for the departure of the satellite's shape from hydrostaticity, by $\sim 1.5 \mathrm{~km}$, even if the outer shell of the satellite is likely to have already relaxed. Topographic features, for example, unrelaxed craters are another source of density anomalies.

A knowledge of the principal axis moment of inertia is key to estimating the departure from hydrostatic equilibrium that is generally assumed to interpret degree-two gravity and oblate shape data in terms of interior properties through simple relationships (Eq. (2), Zharkov et al. 1985). A determination of the mean moment of inertia $I$ from $(A+B+C) / 3$ independently of the former equation by using the rotational motion of the body (see Ferrari et al. 1980; Konopliv et al. 2006) would enable the detection of large variations in internal structure. Unfortunately, the amplitudes of the nutation and the precessional motion of Ceres are very small. Their measurement requires the tracking of a landmark at the surface of Ceres with an accuracy better than $10 \mathrm{~cm}$, and this for at least six months. In addition, since it is unlikely that Ceres' obliquity is fully damped (Sect. 3), we cannot rely on the assumption that Ceres is in the generalized Cassini state as a means of determining its principal axis moments of inertia. Therefore, the comparison of gravity and shape data appears to be the best way of inferring the presence of nonhydrostatic anomalies (with the Chandler mode, if detected) in the case of Ceres.

The extent of global relaxation can also be assessed from the comparison of the secular Love numbers inferred independently of the shape $h_{\mathrm{s}}$ and the degree-two gravity field $k_{\mathrm{s}}$ (e.g. Dermott \& Thomas 1988).

The Love number $k_{\mathrm{s}}$ can be inferred from the degree-two gravity field such as Eq. (2). If the object is in hydrostatic equilibrium, then the Love numbers are related by (e.g., Zharkov et al. 1985)

$h_{\mathrm{s}}=k_{\mathrm{s}}+1$.

Departure from this relationship provides a measure of the nonhydrostaticity of Ceres. The Dawn mission is likely to yield the gravity field of Ceres to degree 10 as an outcome of the nominal mission (Konopliv et al. 2011). The ratio of the gravity data to the topography (admittance) is generally used to constrain the degree of isostatic relaxation achieved by geological features (e.g., Simons et al. 1994). For example, Nimmo et al. (2010) interpreted Rhea's degree-three gravity coefficient inferred by Iess et al. (2007) from the Cassini Orbiter, as the signature of unrelaxed impact craters. Line-of-sight gravity measurements are 
also most appropriate for detecting lateral variations in density, to be compared against the topography measurements inferred from high-resolution imaging.

\section{Conclusion}

We have characterized the main components of Ceres' rotation and quantified them by assuming that Ceres can be differentiated into a rocky core and icy shell. First, our modeling predicts that Ceres' obliquity is not constrained by the dissipative history of the asteroid. However, multiple determinations of Ceres' pole agree that its obliquity lies between 0.01 and $4 \mathrm{deg}$. The lower bound suggests that Ceres could be relaxed to a generalized Cassini state. However, owing to the very long damping timescale, this situation is unlikely. This uncertainty will be resolved by the Dawn mission. In any case, important constraints can also be inferred by combining shape and gravity data. These will yield independent determinations of the secular Love number that will be used to constrain Ceres' hydrostatic state, from which the mean moment of inertia of the asteroid can be inferred.

For the stratified, solid model considered in this study, we have established upper bounds on the rigid and non-rigid components of the nutations, polar motion, and l.o.d. These appear to be too small to be inferred from space measurement techniques. We then identified that a detectable perturbation of Ceres' spin state (wobble) may be the signature of a Chandler mode. This mode would have to be excited by recent large impacts or currents in local liquid reservoirs at depth to yield a sizeable signature. This aspect needs to be quantified in detail as it offers the prospect to constrain Ceres' thermal state and geophysical evolution.

Acknowledgements. The authors wish to thank Jim Williams (JPL) and Richard Gross (JPL) for valuable discussions on the secular orbits of asteroids and the excitation of the Chandler wobble. The authors are also thankful to the anonymous reviewers, who helped improve the quality of this manuscript. Part of this work was conducted at the Jet Propulsion Laboratory, California Institute of Technology, under contract to NASA. Government sponsorship acknowledged. All rights reserved. This research benefits from the financial support of the Paris Observatory (2010).

\section{References}

Bills, B. G., \& Nimmo, F. 2010, Icarus, in press

Carry, B., Dumas, C., Fulchignoni, M., et al. 2008, A\&A, 478, 235

Castillo-Rogez, J. C., \& McCord, T. B. 2010, Icarus, 205, 443

Castillo-Rogez, J. C. 2011, Icarus, in press

Castillo-Rogez, J. C., Efroimsky, M., \& Lainey, V. 2011, J. Geophys. Res., submitted

Colombo, G. 1966, AJ, 71, 891

Correia, A. C. M. 2009, ApJ, 704, L1

Dehant, V., \& Mathews, M. P. 2007, Earth Rotation Variations, in Treatise of Geophysics, invited paper (Elsevier Publ.), "Geodesy”, ed. T. Herring, \& J. Schubert, 3, 295

Dehant, V., de Viron, O., \& Greff-Lefftz, M. 2005, A\&A, 438, 1149

Dehant, V., Folkner, W., \& Renotte, E. 2009, Planet. Space Sci., 57, 1050

Dermott, S. F. \& Thomas, P. C. 1988, Icarus, 73, 25

Dodson-Robinson, S. E., Bodenheimer, P., Laughlin, G., et al. 2008, ApJ, 688, L99

Drummond, J., \& Christou, J. 2008, Icarus, 197, 480

Fanale, F., \& Salvail, J. R. 1989, Icarus, 82, 97

Farinella, P., \& Davis, D. R. 1992, Icarus, 97, 111
Ferrari, A. J., Sinclair, W. S., Sjogren, W. L., Williams, J. G., \& Yoder, C. F. 1980, J. Geophys. Res., 85, 3939

Gauchez, D., \& Souchay, J. 2006, Icarus, 185, 83

Giorgini, J. D., Yeomans, D. K., Chamberlin, A. B., et al. 1996, BAAS, 28, 3, 1158

Greff-Lefftz, M., Legros, H., \& Dehant, V. 2000, Phys. Earth Planet. Inter., 122, 187

Henrard, J., \& Murigande, C. 1987, Celest. Mech. 40, $345 Đ 366$

Hogenboom, D. L., Kargel, J. S., Consolmagno, G. J., et al. 1997, Icarus, 128, 171

Holsapple, K. A. 1993, Ann. Rev. Earth Planet. Sci., 21, 333

Hussmann, H., \& Spohn, T. 2004, Icarus, 171, 391

Iess, L., Rappaport, N. J., Tortora, P., et al. 2007, Icarus, 190, 585

Jedicke, R., Larsen, J., \& Spahr, T. 2002, Observational Selection Effects in Asteroid Surveys, Asteroids III, 71

Konopliv, A. S., Yoder, C. F., Standish, E. M., Yuan, D.-N., \& Sjogren, W. L. 2006, Icarus, 182, 23

Konopliv, A. S., Asmar, S. W., Bills, B. G., et al. 2011, Space Sci. Rev., 78

Koot, L., Dumberry, M., Rivoldini, A., de Viron, O., \& Dehant, V. 2010, Geophys. J. Int., 182, 1279

Lemaitre, A., DHoedt, S., \& Rambaux, N. 2006, Cel. Mech. Dyn. Astron., 95, 213

Levrard, B., Correia, A. C. M., Chabrier, G., et al. 2007, A\&A, 462, L5

Margot, J. L., Peale, S. J., Jurgens, R. F., Slade, M. A., \& Holin, I. V. 2007, Science, 316, 710

Mathews, P. M., Herring, T. A., \& Buffett, B. A. 2002, J. Geophys. Res., 107, 2068

McCarthy, C. M., \& Castillo-Rogez, J. C. 2011, Planetary ices attenuation, Science of Solar System Ices, ed. M. S. Gudipati, \& J. Castillo-Rogez, in press

McCord, T. B., \& Sotin, C. 2005, J. Geophys. Res., 110

McCord, T. B., Castillo-Rogez, J. C., \& Rivkin, A. S. 2011, Space Sci. Rev., in press

Mousis, O., Alibert, Y., Hestroffer, D., et al. 2008, MNRAS, 383, 1269

Millis, R. L., et al. 1987, 72, 507

Moritz, H., \& Mueller, I. I. 1987, Earth Rotation: Theory and Observations (New York: Ungar)

Munk, W. H., \& MacDonald, G. J. F. 1960, The Rotation of the Earth: a Geophysical Discussion (Cambridge [Eng.] University Press)

Néron de Surgy, O., \& Laskar, J. 1997, A\&A, 318, 975

Nimmo, F., \& Matsuyama, I. 2007, Geophys. Res. Lett., 34, L19203

Nimmo, F., Bills, B. G., Thomas, P. C., \& Asmar, S. W. 2010, J. Geophys. Res. (Planets), 115, 10008

Noir, J., Hemmerlin, F., Wicht, J., Baca, S. M., \& Aurnou, J. M. 2009, Phys. Earth Planet. Int., 173, 141

Palguta, J., Schubert, G., Zhang, K., \& Anderson, J. D. 2009, Icarus, 201, 615

Peale, S. J. 1969, AJ, 74, 483

Peale, S. J. 1975, J. Geophys. Res., 80, 4939

Prieto-Ballesteros, O., \& Kargel, J. S. 2005, Icarus, 173, 212

Rivkin, A. S., \& Volquardsen, E. L. 2010, Icarus, 206, 327

Roosbeek, F. 1995, Potentiel de marées, nutations et précession d'une Terre rigide, Ph.D. Thesis

Roosbeek, F., \& Dehant, V. 1998, Cel. Mech. Dyn. Astron., 70, 215

Russell, C. T., Capaccioni, F., Coradini, A., et al. 2007, Earth, Moon, and Planets, 101,65

Schenk, P. M., \& McKinnon, W. B. 2008, 39th Lunar and Planetary Sci. Conf., 2523

Simons, M., Hager, B. H., \& Solomon, S. C. 1994, Science, 264, 798

Takeuchi, H., \& Saito, M. 1972, Seismic surface waves, in Methods in Computational Physics, ed. B. A. Bolt (New York: Academic Press), 1, 217

Thomas, C., Parker, J. Wm., McFadden, L. A., et al. 2005, Nature, 437, 224

Tyler, R. H. 2008, Nature, 456, 770

Tobie, G., Mocquet, A., \& Sotin, C. 2005, Icarus, 177, 534

Van Hoolst, T., Rambaux, N., Karatekin, Ö., Dehant, V., \& Rivoldini, A. 2008, Icarus, 195, 386

Williams, J. G., Boggs, D. H., Yoder, C. F., Ratcliff, T., \& Dickey, J. O. 2001, J. Geophys. Res., 106, 27, 933

Yoder, C. F. 1995, in Global Earth Physics, A Handbook of Physical Constants, AGU Reference Shelf 1

Zharkov, V. N., Leontjev, V. V., \& Kozenko, V. A. 1985, Icarus, 61, 92

Zolotov, M. Yu. 2009, Icarus, 204, 183 